\title{
Asymptotic-Induced Domain Decomposition Methods for Kinetic and Drift Diffusion Semiconductor Equations
}

\author{
by A. KLAR
}

\begin{abstract}
This paper deals with domain decomposition methods for kinetic and drift diffusion semiconductor equations. In particular accurate coupling conditions at the interface between the kinetic and drift diffusion domain are given. The cases of slight and strong nonequilibrium situations at the interface are considered and numerical examples are shown.
\end{abstract}

\section{Introduction}

Semiconductor device simulations are usually done on the basis of drift diffusion or hydrodynamic equations. However in view of the ongoing miniaturization of semiconductor devices a more accurate modelling of the physics seems to be necessary. This is achieved by kinetic semiconductor equations. We refer to the books of Markowich et al. [16] and Selberherr [18] for a detailed description of semiconductor equations and further references. However, it is in general not necessary to model the whole region in the device by the computationally expensive kinetic equation. Only in particular sensitive regions, where the solution is far away from an equilibrium state, as for example in boundary layers, kinetic equations have to be employed. In the remaining regions of the device the usual drift diffusion equation may be valid, and will lead to sufficiently accurate results.

Domain decompositon methods are a natural tool to design in this case accurate numerical codes with reasonable computation times solving both types of equations in their respective domains. This can be achieved similiar to the field of gas dynamics, where domain decomposition methods have lead to the development of hybrid codes for kinetic and hydrodynamic equations. Examples can be found in $[3,15]$.

The general aim is to approximate the global kinetic solution by the solution of the domain decomposition problem, computed with the hybrid code, as accurately as possible, saving in this way a considerable amount of computing 
time. Once the locations of the kinetic and drift diffusion domains in the device are known, the main point of the problem is to obtain the correct coupling conditions at the interface between the two regions.

In the present paper the physics of the semiconductor device is assumed to be described by a kinetic transport equation. We restrict here to kinetic semiconductor equations with a linear collision term and given electric field. However the following analysis should be extendable to nonlinear equations and selfconsistent treatments of the electric field.

In section 2 we state the physical problem and the equations describing it. In section 3 we state coupling conditions for equilibrium situations at the interface. Using the equality of fluxes, a condition that is due to Maxwell in the gas dynamics case and to Marshak in the radiative transfer case, or simply the equality of moments at the interface and an $O\left(\epsilon^{2}\right)$ approximation of the distribution function, where $\epsilon$ is the mean free path, one obtains correct results. These conditions are proven to yield $O(\epsilon)$ approximations for a model situation. In section 4 nonequilibrium situations at the interface are considered. Using asymptotic analysis similiar to the usual boundary layer considerations as, e.g., in Bardos et al. [1] and Bensoussan et al. [2] for neutron transport, Cercignani [4] and Sone and coworkers [19] in the gas dynamics case and Poupaud [17], Golse/Poupaud [8] and Yamnahakki [20] for semiconductor equations, we develop accurate coupling conditions in this case by an analysis of the interface layer between the two domains. This leads to kinetic linear half space problem. The essential point is then to find a fast approximate solution procedure of the half space problem. This will be discussed in the appendix. It is an extension of the work done in Golse/Klar [7] and related to the so called variational methods, see Loyalka [14] and Golse [6] for gas dynamics and Larsen, Pomraning [13] in the radiative transfer case. In section 5 we show some numerical results for a one dimensional model problem. As one can see in the simulations the developed coupling conditions lead to a very accurate approximation of the kinetic solution by the solution of the domain decomposition problem.

\section{The Basic Equations}

We consider a domain $D_{s}$ in $\mathbb{R}^{3}$ representing the semiconductor device geometry. $D_{s}$ is divided into two not overlapping subdomains $D_{1}$ and $D_{2}, D_{1} \cup D_{2}=D_{s}$ with smooth boundaries $\partial D_{s}, \partial D_{1}, \partial D_{2}$ and the interface $I=\partial D_{1} \cap \partial D_{2}$.

The basic description of the physics in the device is given by the following linear semiconductor Boltzmann equations in $D_{i}, i=1,2$ with mean free paths $\epsilon_{i}$

$$
\partial_{t} f_{i}+\frac{1}{\epsilon_{i}}\left(v \cdot \nabla_{x} f_{i}+E \cdot \nabla_{v} f_{i}\right)+\frac{1}{\epsilon_{i}^{2}} Q\left(f_{i}\right)=S M,
$$

where $f_{i}=f_{i}(x, v, t)$ are the distribution functions of the electrons in $D_{i}, x \in$ $D_{i}, v \in \mathbb{R}^{3}, t \geq 0$. See e.g. Markowich et al. [16] for a discussion of these equations. 
$E=E(x, t)$ is the electric field multiplied by the absolute value of the elementary charge and divided by the effective mass.

$$
Q\left(f_{i}\right)(v)=\int_{\mathbb{R}^{3}} \Phi(v, w)\left(M(w) f_{i}(v)-M(v) f_{i}(w)\right) d w
$$

is the collision operator with

$$
\Phi(-v,-w)=\Phi(v, w)=\Phi(w, v)>0
$$

and

$$
M(v)=\frac{1}{(2 \pi \theta)^{\frac{3}{2}}} \exp \left(-\frac{|v|^{2}}{2 \theta}\right),
$$

where $\theta$ is the temperature of the device. $S=S(x, t)$ models the generation recombination process. Defining

$$
\lambda(v)=\int \Phi(v, w) M(w) d w
$$

and

$$
K\left(f_{i}\right)(v)=\int \Phi(v, w) f_{i}(w) d w
$$

we get

$$
Q\left(f_{i}\right)(v)=\lambda(v) f_{i}(v)-M(v) K\left(f_{i}\right)(v) .
$$

The global kinetic solution is then defined by the solution of equations (1) in $D_{i}$ with the initial and boundary conditions

$$
f_{1}(x, v, 0)=g(x, v) M(v), x \in D_{1}
$$

and

$$
f_{1}(x, v, t)=f_{B}(x, v, t) M(v), x \in \partial D_{s} \cap \partial D_{1}, v \cdot n(x)<0,
$$

where $n(x)$ is the outer normal at $\partial D_{s}$ at the point $x$ and

$$
f_{2}(x, v, 0)=g(x) M(v), x \in D_{2}
$$

and

$$
f_{2}(x, v, t)=f_{B}(x, t) M(v), x \in \partial D_{s} \cap \partial D_{2}, v \cdot n(x)<0 .
$$

The interface condition is simply continuity of the distribution functions for $x \in I$

$$
f_{1}(x, v, t)=f_{2}(x, v, t) .
$$

The mean free path $\epsilon_{2}=\epsilon$ in $D_{2}$ is assumed to be small such that an approximation of the kinetic equation by the drift diffusion equation is valid there, see, e.g., Poupaud [17] for a mathematical treatment.

This means, $f_{2}(x, v, t)$ is approximated by $\rho(x, t) M(v)$, where $\rho(x, t)$ fulfills

$$
\partial_{t} \rho+\nabla_{x} \cdot\left(-D \nabla_{x} \rho+\mu E \rho\right)=S,
$$


with the diffusion coefficient

$$
D=\int v_{i} h_{i}(v) d v, i=1,2,3,
$$

where $h_{i}$ is the solution of

$$
Q\left(h_{i}\right)=v_{i} M(v), \int h_{i}(v) \lambda(v) d v=0
$$

and the mobility

$$
\mu=D \theta \text {. }
$$

We set in the following $\theta=1$, i.e. $\nu=D$ to simplify the notation.

The aim is now to approximate the global kinetic solution by the solution of the following coupling problem: In $D_{1}$ the kinetic equation (1) is solved with the above inital conditions in $D_{1}$ and the boundary condition at $\partial D_{1} \cap \partial D_{s}$. In $D_{2}$ the drift diffusion equation (2) is solved with initial conditions

$$
\rho(x, 0)=g(x), x \in D_{2}
$$

and boundary conditions

$$
\rho(x, t)=f_{B}(x, t), x \in \partial D_{2} \cap \partial D_{s}
$$

Equiping these equations with coupling conditions at the interface I will lead to a properly stated problem. The solution depends however strongly on the type of coupling conditions employed. In the next sections we discuss several possibilities and investigate them.

\section{Coupling Conditions for Near Equilibrium States}

For equilibrium states at the interface coupling conditions can be straightforwardly obtained. One starts by defining a 'macroscopic distribution function' approximating the kinetic distribution function in $D_{2}$ to $O\left(\epsilon^{2}\right)$. Using an approximation to $O(\epsilon)$ leads without overlapping of the domains to obviously wrong results, see section 5, Figure 1.

Assuming, as in $[1,2,17]$, that $f_{2}$ in $D_{2}$ is of the form

$$
f_{2}^{(0)}+\epsilon f_{2}^{(1)}+\cdots
$$

with $\epsilon=\epsilon_{2}$ and substituting it into the scaled equations (1) leads to

$$
\begin{array}{r}
Q\left(f_{2}^{(0)}\right)=0 \\
Q\left(f_{2}^{(1)}\right)=-\left(v \cdot \nabla_{x} f_{2}^{(0)}+E \cdot \nabla_{v} f_{2}^{(0)}\right)
\end{array}
$$

Therefore due to (3) and due to the properties of the collision kernel, see, e.g., Poupaud [17] we obtain

$$
f_{2}^{(0)}(x, v, t)=\rho(x, t) M(v)
$$


This means that the $O(\epsilon)$ macroscopic distribution function would be given for $x \in D_{2}$ by

$$
f_{\text {macro }}(x, v, t)=\rho(x, t) M(v)
$$

(4) leads to

$$
f_{2}^{(1)}(x, v, t)=-\epsilon h(v) \cdot\left(\nabla_{x} \rho(x, t)-E(x, t) \rho(x, t)\right) .
$$

Therefore we get for $x \in D_{2}$

$$
f_{\text {macro }}(x, v, t)=\rho(x, t) M(v)-\epsilon h(v) \cdot\left(\nabla_{x} \rho-E(x, t) \rho\right) .
$$

as the $O\left(\epsilon^{2}\right)$ macroscopic distribution function in $D_{2}$. Using the latter one, one obtains the following conditions:

\section{The equality of moments:}

Determine $\rho(\hat{x}, t), \hat{x} \in I$ by

$$
\begin{aligned}
\int f_{1}(\hat{x}, v, t) d v & =\int f_{\text {macro }}(\hat{x}, v, t) d v \\
& =\int \rho(\hat{x}, t) M(v)-\epsilon h(v) \cdot\left(\nabla_{x} \rho(\hat{x}, t)-E(\hat{x}, t) \rho(\hat{x}, t)\right) d v .
\end{aligned}
$$

The ingoing function for the kinetic region is given by

$$
f_{1}(\hat{x}, v, t)=f_{\text {macro }}(\hat{x}, v, t)=\rho(\hat{x}, t) M(v)-\epsilon h(v) \cdot\left(\nabla_{x} \rho(\hat{x}, t)-E(\hat{x}, t) \rho(\hat{x}, t)\right)
$$

for $\hat{x} \in I, v \cdot n<0$, where $n=n(\hat{x})$ is the outer normal at $\partial D_{1}$ at $\hat{x}$.

\section{The Maxwell-Marshak conditions:}

Determine $\rho(\hat{x}, t), \hat{x} \in I$ by

$$
\begin{aligned}
& \int_{v \cdot n>0} v \cdot n f_{1}(\hat{x}, v, t) d v=\int_{v \cdot n>0} v \cdot n f_{\text {macro }}(\hat{x}, v, t) d v \\
= & \int_{v \cdot n>0} v \cdot n\left[\rho(\hat{x}, t) M(v)-\epsilon h(v) \cdot\left(\nabla_{x} \rho(\hat{x}, t)-E(\hat{x}, t) \rho(\hat{x}, t)\right)\right] d v .
\end{aligned}
$$

with $n=n(\hat{x})$. The ingoing function for the kinetic region is given as before.

The solution of equation (1) in $D_{1}$ and (2) in $D_{2}$ with the initial, boundary and coupling conditions as above will be called the solution of the coupling problem. It can be found numerically by a standard Schwarz type iteration procedure: One determines the coupled solution by solving in turn the equations in the two domains. In each step the above interface conditions are used. This is repeated until a stationary state is obtained. See Klar [12] for an investigation of the coupled solution and of the iteration procedure in a general case.

In the following we show for a model situation that these coupling conditions lead to a correct approximation in case of near equilibrium situations.

We consider the stationary kinetic equations

$$
\frac{1}{\epsilon_{i}}\left(v \cdot \nabla_{x} f_{i}+E \cdot \nabla_{v} f_{i}\right)+\frac{1}{\epsilon_{i}^{2}} Q\left(f_{i}\right)=S M,
$$


with $x \in D_{i}, i=1,2$ The mean free paths are $\epsilon_{1}=\epsilon_{2}=\epsilon$. The stationary drift diffusion equation is for $\theta=1$

$$
\nabla_{x} \cdot\left(-\nabla_{x} \rho+E \rho\right)=\frac{S}{D}
$$

with $x \in D_{2}$. We restrict to a slab $x \in D_{s}=[A, B], A<0, B>0$. The subdomains are $D_{1}=[A, 0], D_{2}=[0, B]$. The boundary conditions are $f(A, v)=$ $\rho_{A} M(v), v_{1}>0, \rho(B)=\rho_{B} M(v), v_{1}<0$. The electric field is of the form $E(x)=\left(E_{1}(x), 0,0\right)$. Equation (5) in $D_{1}$ is coupled to equation (6) in $D_{2}$ by one of the above coupling conditions. $f_{1}$ and $\rho$ denote the coupled solution in the respective domains. The reference solution is the global stationary kinetic solution, i.e. the solutions $f_{1}, f_{2}$ of the kinetic equation (5) in $D_{1}$ and $D_{2}$ with $f_{1}(0, v, t)=f_{2}(0, v, t)$. We denote the global solution by $f(x, v, t), x \in D_{s}$. One can then prove:

\section{Theorem 1}

The solution of the coupling problem for the stationary equations (5) and (6) with one of the above coupling conditions at the interface is an $O(\epsilon)$ approximation of the global stationary kinetic solution in the whole domain, i.e.

$$
\begin{aligned}
\left|f_{1}(x, v, t)-f(x, v, t)\right| & \leq C \epsilon, x \in D_{1} \\
|\rho(x, t)-f(x, v, t)| & \leq C \epsilon, x \in D_{2}
\end{aligned}
$$

where $C$ is some constant.

We need a trivial lemma:

\section{Lemma 1}

Let $\rho(x)$ be the solution of the drift diffusion equation $(6)$ in $D_{2}=[0, B]$ with the Dirichlet boundary data $\rho(0)$ at $x=0$ and $\rho_{B}$ at $x=B$. Then the value $\partial_{x} \rho(0)$ is given by

$$
\partial_{x} \rho(0)=\rho(0)\left(E_{1}(0)-G\right)+H
$$

with

$$
G=\left(\int_{0}^{B} e^{-\bar{E}\left(x^{\prime}\right)} d x^{\prime}\right)^{-1}, \bar{E}(x)=\int_{0}^{x} E_{1}\left(x^{\prime}\right) d x^{\prime}
$$

and

$$
H=G \rho_{B} e^{-\bar{E}(B)}+G \int_{0}^{B} e^{-\bar{E}\left(x^{\prime}\right)} \bar{S}\left(x^{\prime}\right) d x^{\prime}, \bar{S}(x)=\frac{1}{D} \int_{0}^{x} S\left(x^{\prime}\right) d x^{\prime}
$$

Proof of the Theorem:

We restrict to the Maxwell-Marshak conditions. They give together with the Lemma

$$
\int_{v_{1}>0} v_{1} f_{1}(0, v) d v=\rho(0) C+\epsilon Z(\rho(0) G-H)
$$


with

$$
C=\int_{v_{1}>0} v_{1} M(v) d v, Z=\int_{v_{1}>0} v_{1} h_{1}(v) d v
$$

and for $v_{1}<0$

$$
\begin{aligned}
f_{1}(0, v) & =\rho(0) M(v)-\epsilon h_{1}(v)\left(\partial_{x} \rho(0)-E_{1} \rho(0)\right) \\
& =\rho(0)\left(M(v)+\epsilon h_{1}(v) G\right)-\epsilon h_{1}(v) H
\end{aligned}
$$

From (7) we obtain

$$
\rho(0)=(C+\epsilon G Z)^{-1}\left(\int_{v_{1}>0} v_{1} f_{1}(0, v) d v+\epsilon Z H\right) .
$$

Substituting this into (8) a short calculation gives for $v_{1}<0$ the following reflecting boundary condition for the kinetic part of the coupled solution

$f_{1}(0, v)=\epsilon f_{1}(0, v) b(v)+\int_{v_{1}^{\prime}>0}\left(B_{1}\left(v, v^{\prime}\right)+\epsilon B_{2}\left(v, v^{\prime}\right)\right) f_{1}\left(0, v^{\prime}\right) d v^{\prime}+\epsilon g(v)+O\left(\epsilon^{2}\right)$

with

$$
\begin{array}{r}
b(v)=-G \frac{Z}{C}, \\
B_{1}\left(v, v^{\prime}\right)=v_{1}^{\prime} \frac{1}{C} M(v), \\
B_{2}\left(v, v^{\prime}\right)=v_{1}^{\prime} \frac{G}{C} h_{1}(v), \\
g(v)=\left(\frac{Z}{C} M(v)-h_{1}(v)\right) H
\end{array}
$$

Considering now the kinetic equation in $D_{1}$ with the fixed boundary conditions $\rho_{A}$ at $A$ and the above reflecting boundary condition at 0 , we observe that the approximation of this kinetic problem for small $\epsilon$ by a drift diffusion equation is similar to a problem in neutron transport, that has been treated in Bensoussan et al. [2], section 3.6.

A straightforward extension of the results obtained there leads to an $O(\epsilon)$ approximation of the kinetic solution $f_{1}$ in $D_{1}$ with the above reflecting boundary conditions at 0 by a drift diffusion equation with the following boundary condition at $x=0$ :

$$
\bar{B}_{1} \partial_{x} \rho(0)+\left(\bar{B}_{2}+\bar{b}\right) \rho(0)-\bar{B}_{1} E_{1}(0) \rho(0)+\bar{g}=0
$$

with

$$
\begin{aligned}
\bar{B}_{1} & =-\int_{v_{1}<0} \frac{v_{1}}{C}\left[Q^{-1}\left(v_{1} M(v)\right)-\int_{v_{1}^{\prime}>0} B_{1}\left(v, v^{\prime}\right) Q^{-1}\left(v_{1}^{\prime} M\left(v^{\prime}\right)\right) d v^{\prime}\right] d v \\
& =-\int_{v_{1}<0} \frac{v_{1}}{C}\left[h_{1}(v)-\frac{1}{C} \int_{v_{1}^{\prime}>0} v_{1}^{\prime} M(v) h_{1}\left(v^{\prime}\right) d v^{\prime}\right] d v \\
& =-\frac{1}{C} \int v_{1} h_{1}(v) d v
\end{aligned}
$$




$$
\begin{aligned}
\bar{B}_{2} & =-\int_{v_{1}<0} \frac{v_{1}}{C} \int_{v_{1}^{\prime}>0} M\left(v^{\prime}\right) B_{2}\left(v, v^{\prime}\right) d v^{\prime} d v \\
& =\int_{v_{1}<0} v_{1} h_{1}(v) d v \frac{G}{C}, \\
\bar{g} & =-\int_{v_{1}<0} \frac{v_{1}}{C} g(v) d v=-\int_{v_{1}<0} \frac{v_{1}}{C}\left[\frac{Z}{C} M(v)-h_{1}(v)\right] d v H \\
& =\left[\frac{Z}{C}+\frac{1}{C} \int_{v_{1}<0} v_{1} h_{1}(v) d v\right] H=\frac{H}{C} \int v_{1} h_{1}(v) d v, \\
\bar{b} & =-\int_{v_{1}<0} \frac{v_{1}}{C} b(v) M(v) d v=-G \frac{Z}{C} \int_{v_{1}<0} \frac{v_{1}}{C} M(v) d v=G \frac{Z}{C} .
\end{aligned}
$$

This gives

$$
\partial_{x} \rho(0)-\rho(0)\left(E_{1}(0)-G\right)-H=0 .
$$

as the boundary condition for a drift diffusion approximation of $f_{1}$ in $D_{1}$.

Considering now the global kinetic solution in $[A, B]$ we know that it is approximated to $O(\epsilon)$ by the global drift diffusion equation, see [17]. However, the solution of the global drift diffusion equation in $D_{s}$ is in $D_{1}$ equal to the solution of the drift diffusion equation in $D_{1}$ with the above boundary condition at 0 . This can be seen by an easy calculation.

Therefore finally we get an $O(\epsilon)$ approximation of the global kinetic solution by the coupled solution in $D_{1}$. The corresponding property in $D_{2}$ is then obvious.

This result shows, that the equality of moments or fluxes is appropriate for near equilibrium situations at the interface. If this is not the case, they might give wrong results as shown in section 5 .

In the next section we develop accurate however easy to handle coupling conditions for this case.

\section{Coupling Conditions for Nonequilibrium States}

In contrast to section 3 non equilibrium situations might prevail here at the interface between the two domains. We use here not only an $O\left(\epsilon^{2}\right)$ approximation in the bulk of the device, but also on the boundaries and interfaces to develop the correct conditions. This includes an analysis of the interface layer between the two domains. We concentrate in this section on the interface layer neglecting the boundary layers.

Proceeding similarly as in the usual boundary layer treatment, see the references in the introduction, one assumes the macroscopic distribution function in $D_{2}$ to be the same as in the last section plus an additional interface layer term 
concentrated around the interface I with a thickness of the order of a mean free path:

$$
\begin{aligned}
& f_{\text {macro }}(x, v, t) \\
= & \rho(x, t) M(v)-\epsilon h(v) \cdot\left(\nabla_{x} \rho(x, t)-E(x, t) \rho(x, t)\right)+\chi\left(\frac{y^{\prime}}{\epsilon}, \alpha_{i}, v, t\right) \\
+ & \epsilon \hat{\chi}\left(\frac{y^{\prime}}{\epsilon}, \alpha_{i}, v, t\right)+\epsilon^{2} W(x, v, t)+\epsilon^{2} \hat{W}\left(\frac{y}{\epsilon}, \alpha_{i}, v, t\right),
\end{aligned}
$$

where $x \in D_{2} . y$ and $\alpha_{i}, i=1,2$ are interface layer coordinates given by the following: Let $\hat{x}$ be the point on the interface closest to $x$. The coordinate along the normal to the interface is $y^{\prime}$, i.e. $x=\hat{x}+n(\hat{x}) y^{\prime}$, where $n(\hat{x})$ is the outer normal at $\partial D_{1}$ at $\hat{x} \in I$. The interface itself is parametrized by $\alpha_{i}$, i.e. $\hat{x}=\hat{x}\left(\alpha_{1}, \alpha_{2}\right)$, where the $\alpha_{i}$ depend on the point $x$. See, e.g., [4],[19] for details on boundary layer coordinates.

Requiring that the kinetic equation in $D_{2}$ is fulfilled by $f_{\text {macro }}$ to $O\left(\epsilon^{2}\right)$, i.e.,

$$
\begin{array}{r}
\epsilon \partial_{t} f_{\text {macro }}+\left(v \cdot \nabla_{x} f_{\text {macro }}+E(x, t) \cdot \nabla_{v} f_{\text {macro }}\right)+\frac{1}{\epsilon} Q\left(f_{\text {macro }}\right) \\
=\epsilon S M+O\left(\epsilon^{2}\right),
\end{array}
$$

we obtain the following equations by substituting $f_{\text {macro }}$ from (9) into (10) and comparing terms of the same order in $\epsilon$ :

Up to order $\epsilon^{-} 1$ we get the following halfspace problem at $\hat{x}$

$$
v \cdot n \partial_{y} \chi+Q(\chi)=0 .
$$

with $\chi=\chi\left(y, \alpha_{i}, v, t\right), y \in[0, \infty)$ and $n=n(\hat{x})$. To order 1 we obtain

$$
\begin{array}{r}
v \cdot \nabla_{x}(\rho(x, t) M(v))+E(x, t) \cdot \nabla_{v}(\rho M) \\
=Q\left(h(v) \cdot\left[\nabla_{x} \rho(x, t)-E(x, t) \rho(x, t)\right]\right)
\end{array}
$$

and another halfspace problem at the interface point $\hat{x}$ with $y \in[0, \infty)$ :

$$
v \cdot n \partial_{y} \hat{\chi}+E(\hat{x}, t) \nabla_{v} \chi+\sum_{i=1}^{2} v \cdot \nabla_{x} \alpha_{i} \partial_{\alpha_{i}} \chi+Q(\hat{\chi})=0 .
$$

Remark that equation (12) is fulfilled since $Q(h)=v M(v)$.

To order $\epsilon$ we must have

$$
\partial_{t}(\rho M)-v \nabla_{x}\left(h\left(\nabla_{x} \rho-E \rho\right)\right)-E \nabla_{v}\left(h\left(\nabla_{x} \rho-E \rho\right)\right)+Q(W)=S M
$$

and a linear half space problem for $\hat{W}$ must be fulfilled. Equation (14) is solvable, if the drift diffusion equation is fulfilled by $\rho(x, t)$.

Assuming continuity of the distribution functions at the interface to $O\left(\epsilon^{2}\right)$, i.e., for $\hat{x} \in I$

$$
f_{1}(\hat{x}, v, t)=f_{\text {macro }}(\hat{x}, v, t)+O\left(\epsilon^{2}\right),
$$


we obtain from (9) that the sum of the ingoing functions for the half space problems for $v \cdot n>0$ has to fulfill

$$
\begin{array}{r}
\chi\left(0, \alpha_{i}, v, t\right)+\epsilon \hat{\chi}\left(0, \alpha_{i}, v, t\right) \\
f_{1}(\hat{x}, v, t)-\rho(\hat{x}, t) M(v)+\epsilon h(v) \cdot\left(\nabla_{x} \rho(\hat{x}, t)-E(\hat{x}, t) \cdot \rho(\hat{x}, t)\right)+O\left(\epsilon^{2}\right)
\end{array}
$$

The splitting of this sum into $\chi\left(0, \alpha_{i}, v, t\right)$ and $\hat{\chi}\left(0, \alpha_{i}, v, t\right)$ is arbitrary. We choose,

$\chi\left(0, \alpha_{i}, v, t\right)=f_{1}(\hat{x}, v, t)-\rho(\hat{x}, t) M(v)+\epsilon h(v) \cdot\left(\nabla_{x} \rho(\hat{x}, t)-E(\hat{x}, t) \cdot \rho(\hat{x}, t)\right)$

and

$$
\hat{\chi}\left(0, \alpha_{i}, v, t\right)=0
$$

for $v \cdot n>0$, since the following becomes simpler for this choice. Moreover, the asymptotic values of the halfspace problems must fulfill

$$
\chi\left(\infty, \alpha_{i}, v, t\right)+\epsilon \hat{\chi}\left(\infty, \alpha_{i}, v, t\right)=0,
$$

since the interface layer term should vanish in the bulk.

We introduce two more notations:

Let $\varphi\left(y, \alpha_{i}, v\right)$ be the solution of the half space problem (11) at $\hat{x}$ with

$$
\varphi\left(0, \alpha_{i}, v\right)=k(v)
$$

for $v \cdot n>0$. Then we define the asymptotic value

$$
L_{\hat{x}}(k(\cdot))=\varphi\left(\infty, \alpha_{i}, v\right) M^{-1}(v)
$$

which is independent of $v$ and the Albedo operator

$$
A_{\hat{x}}\left(\left.k(\cdot)\right|_{v \cdot n>0}\right)(v)=\varphi\left(0, \alpha_{i}, v\right)
$$

for $v \cdot n<0$.

Let $\tilde{\chi}\left(y, \alpha_{i}, v, t\right)$ now be the solution of the half space problem (11) at the interface point $\hat{x}$ with ingoing function

$$
\tilde{\chi}\left(0, \alpha_{i}, v, t\right)=f_{1}(\hat{x}, v, t)
$$

for $v \cdot n>0$. From (16) and (17) we have

$$
\begin{array}{r}
\chi\left(\infty, \alpha_{i}, v, t\right)=\tilde{\chi}\left(\infty, \alpha_{i}, v, t\right) \\
-\rho(\hat{x}, t) M(v)+\epsilon L_{\hat{x}}(h(\cdot)) \cdot\left[\nabla_{x} \rho(\hat{x}, t)-E(\hat{x}, t) \rho(\hat{x}, t)\right] M(v)
\end{array}
$$

From $\chi\left(\infty, \alpha_{i}, v, t\right)+\epsilon \hat{\chi}\left(\infty, \alpha_{i}, v, t\right)=0$ we obtain

$$
\begin{array}{r}
-\epsilon \hat{\chi}\left(\infty, \alpha_{i}, v, t\right)=\tilde{\chi}\left(\infty, \alpha_{i}, v, t\right) \\
-\rho(\hat{x}, t) M(v)+\epsilon L_{\hat{x}}(h(\cdot)) \cdot\left[\nabla_{x} \rho(\hat{x}, t)-E(\hat{x}, t) \rho(\hat{x}, t)\right] M(v)
\end{array}
$$


This means, $\rho(\hat{x}, t), \hat{x} \in I$ is determined by the condition

$$
\begin{aligned}
\rho(\hat{x}, t) & =L_{\hat{x}}\left(f_{1}(\hat{x}, \cdot, t)\right)+\epsilon L_{\hat{x}}(h(\cdot)) \cdot\left[\nabla_{x} \rho(\hat{x}, t)-E(\hat{x}, t) \rho(\hat{x}, t)\right] \\
& +\epsilon \hat{\chi}\left(\infty, \alpha_{i}, v, t\right) M^{-1}(v) .
\end{aligned}
$$

Knowing $f_{1}, v \cdot n>0$ this is an equation for $\rho(\hat{x}, t)$. Looking at equation (9) and keeping equation (15) in mind one finds the ingoing function for the Boltzmann region for $\hat{x} \in I$ and $v \cdot n<0$

$$
\begin{aligned}
f_{1}(\hat{x}, v, t)= & \rho(\hat{x}, t) M(v)-\epsilon h(v) \cdot\left(\nabla_{x} \rho(\hat{x}, t)-E(\hat{x}, t) \rho(\hat{x}, t)\right) \\
& +\chi\left(0, \alpha_{i}, v, t\right)+\epsilon \hat{\chi}\left(0, \alpha_{i}, v, t\right) \\
= & A_{\hat{x}}\left(\left.f_{1}(\hat{x}, \cdot, t)\right|_{v \cdot n>0}\right)(v)-\epsilon\left[h(v)-A_{\hat{x}}\left(\left.h(\cdot)\right|_{v \cdot n>0}\right)(v)\right] \\
& \cdot\left(\nabla_{x} \rho(\hat{x}, t)-E(\hat{x}, t) \rho(\hat{x}, t)\right)+\epsilon \hat{\chi}\left(0, \alpha_{i}, v, t\right)
\end{aligned}
$$

The second equality is due to (16) and the definition of the Albedo operator. Knowing $\rho$ this is an equation for $f_{1}$ giving $f_{1}, v \cdot n<$ in terms of $f_{1}, v \cdot n>0$. It is easily handeled in the framework of the Schwarz iteration procedure mentioned in the last section.

It is now most important to have a fast however accurate enough solution procedure to find the asymptotic state and the outgoing distribution of the half space problem. We remark, that solving the whole half space problem, for example, by a standard discretization procedure would need a lot of computing time, in particular, since it has to be solved at each point of the interface. In the appendix this will be achieved by a generalization of a procedure, we developed in Golse/Klar [7], leading to easy to evaluate, however accurate coupling conditions. These conditions yield a considerable improvement compared to the ones obtained by equalizing moments or fluxes as in section 3 .

We mention that in Golse [5] and Klar [11] coupling conditions for the coupling of the Euler equations to the Boltzmann equation have been developed and investigated that are also based on the analysis of a kinetic half space problem. A different approach can be found in Illner/Neunzert [10].

Another remark concerns the situation, when the kinetic domain shrinks to 0 and only the drift diffusion equation is solved in the whole domain. In this case the outgoing function is no longer needed and the condition on $\rho$ becomes a standard slip boundary condition. An extensive treatment of such boundary conditons for the gas dynamic case is given in the work of [18]. Here we mention also the work of [20] on second order boundary conditions for the drift diffusion equation. Using these boundary conditions one obtains a good quality of approximation in the bulk of the device, but obviously no resolution of the solution in the kinetic regions.

Finally, if higher order conditions are required, one may go to higher order in the asymptotic expansion. However, this is very tedious and in most cases it will not be necessary due to other limitations on the accuracy of the solution. 


\section{$5 \quad$ Numerical Results}

In this section we investigate the coupling condition proposed in section $4 \mathrm{nu}-$ merically and compare them to the ones obtained by the equality of moments or fluxes. The equality of moments or fluxes is straightforwardly implemented.

The coupling conditions based on the half space analysis are approximated by the following:

$$
\begin{array}{r}
\rho(\hat{x}, t)=L_{\hat{x}}^{a}\left(f_{1}(\hat{x}, \cdot, t)\right) \\
+\epsilon L_{\hat{x}}^{a}(h(\cdot)) \cdot\left(\nabla_{x} \rho(\hat{x}, t)-E(\hat{x}, t) \cdot \rho(\hat{x}, t)\right)+\epsilon \hat{\chi}^{a}\left(\infty, \alpha_{i}, v, t\right) M^{-1}(v),
\end{array}
$$

where $L_{\hat{x}}^{a}(k(\cdot))$ is defined in (27) for arbitrary $k$ and $\hat{\chi}^{a}(\infty, v, t)$ is given by formula (33).

The equation for the ingoing function for the Boltzmann region $D_{1}$ is explicitely for $v \cdot n<0$

$$
\begin{array}{r}
f_{1}(\hat{x}, v, t)=A_{\hat{x}}^{a}\left(\left.f_{1}(\hat{x}, \cdot, t)\right|_{v \cdot n>0}\right)(v)-\epsilon\left[h(v)-A_{\hat{x}}^{a}\left(\left.h(\cdot)\right|_{v \cdot n>0}\right)(v)\right] \\
\cdot\left(\nabla_{x} \rho(\hat{x}, t)-E(\hat{x}, t) \rho(\hat{x}, t)\right)+\epsilon \hat{\chi}^{a}\left(0, \alpha_{i}, v, t\right)
\end{array}
$$

where $A_{\hat{x}}^{a}\left(\left.k(\cdot)\right|_{v \cdot n>0}\right)$ is determined by (29) for arbitrary $k$ and $\hat{\chi}^{a}\left(0, \alpha_{i}, v, t\right)$ can be approximated by 0 .

In the following figures we calculated two examples. The solutions of the kinetic and drift diffusion equations are determined by a scheme using a straightforward explicit upwind discretization. To find the coupled solution we used, as mentioned before, a Schwarz iteration procedure solving in turn the equations in the kinetic and drift diffusion domain.

The kinetic equation is equation (1) in the slab $D_{s}=[0,1]$. We have choosen a relaxation model for the collision kernel,i.e. $\Phi=1$. The electric field $E(x, t)$ was choosen equal to $\left(E_{1}, 0,0\right)$, where $E_{1} \in \mathbb{R}_{+}$and $S=0$. The density of the electrons is shown for a fixed time $\mathrm{T}$ such that a stationary state is obtained.

In Figure 1 we consider an equilibrium situation. We take $E_{1}=3$. The boundary conditions are $f_{1}(0, v, t)=1, v_{1}>0$ and $f_{2}(1, v, t)=2, v_{1}<0$. The interface is choosen at $x=0.5$.

We show the global kinetic solution, i.e. the reference solution, with mean free paths $\epsilon_{1}=0.01=\epsilon_{2}$. Moreover the solution of the coupling problem is shown, i.e., the solution of the kinetic equation with $\epsilon_{1}$ in $D_{1}$ and the solution of the drift diffusion equation in $D_{2}$. They are coupled together with the Maxwell-Marshak condition using a first and second order approximation of the distribution function in $D_{2}$ as explained in section 2 . The boundary conditions at $x=1$ for the drift diffusion equation are also found by the Maxwell Marshak approximation at the boundary using an asymptotic expansion up to the corresponding order. 
Obviously the first order approximation is completely wrong as was to be expected. The other types of coupling conditions, i.e. the equality of moments and the halfspace conditions, do in this case not differ very much from the Maxwell Marshak conditions.

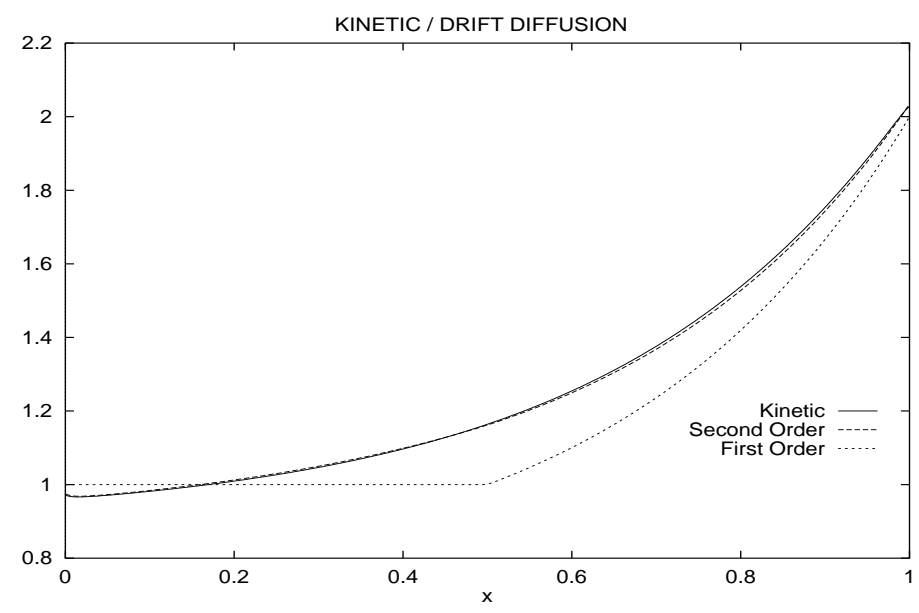

Figure 1: Equilibrium situation - Maxwell-Marshak conditions

In Figure 2 the same kinetic equation is shown but the boundary condition at $x=0$ is $f_{1}(0, v, t)=v_{1}, v_{1}>0 . E_{1}$ was again choosen as $E_{1}=3$. The interface between kinetic and aerodynamic domain is here located at $x=0.02$. The mean free paths are $\epsilon_{1}=0.01=\epsilon_{2}$. All three types of coupling conditions at the interface are shown. The boundary conditions at $x=1$ for the drift diffusion equation are derived by the procedure corresponding to the one used for the coupling condition. In this situation a nonequilibrium situation prevails at the interface. The advantage of a more exact analysis at the interface is clearly seen.

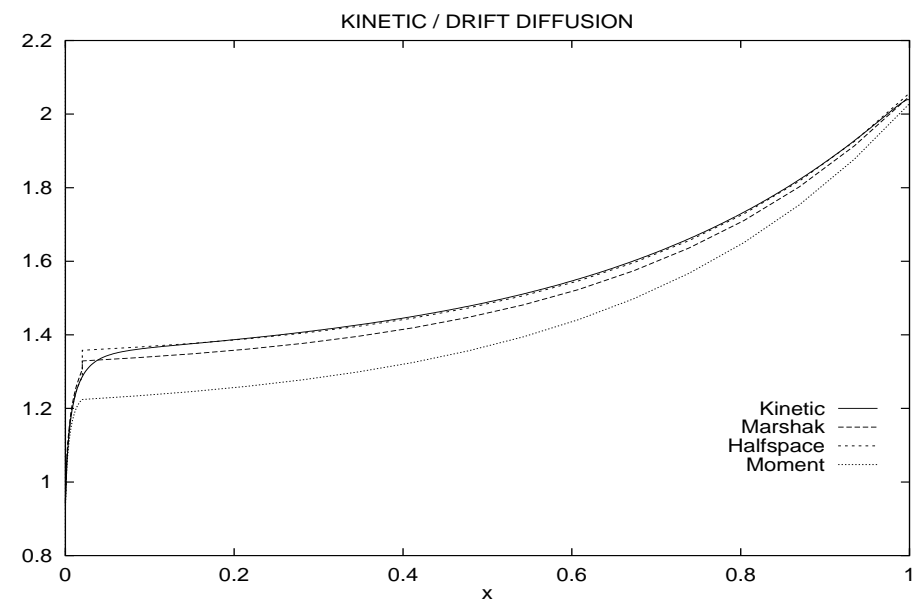

Figure 2: Nonequilibrium situation - Comparison of different coupling conditions 
In Figure 3 the same as in Figure 2 is shown however we plotted only the region around the interface.

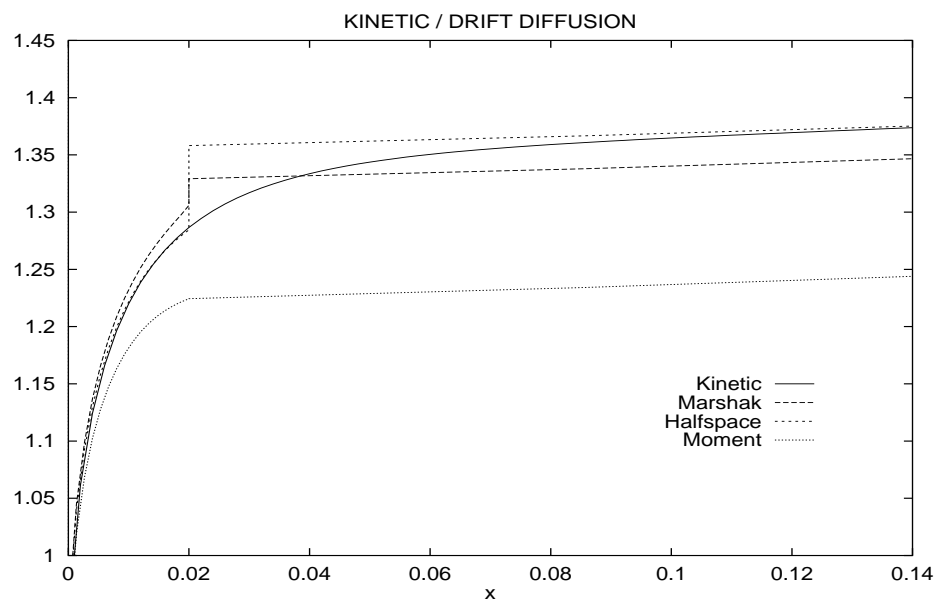

Figure 3: Nonequilibrium situation - Interface region

\section{Appendix: Approximate Solutions of Linear Half Space Problems}

In this section we derive approximations for the asymptotic values and outgoing distributions of the halfspace problem (11) with arbitrary ingoing function and of problem (13) with ingoing function 0 .

Approximation of the solutions of (11):

The equation under consideration is

$$
\begin{aligned}
v \cdot n \partial_{y} \varphi+Q(\varphi) & =0 \\
\varphi\left(0, \alpha_{i}, v\right) & =k(v), v \cdot n>0
\end{aligned}
$$

with

$$
Q(\varphi)(v)=\lambda(v) \varphi(v)-M(v) \int \Phi(v, w) \varphi(w) d w
$$

$y \in[0, \infty)$ and $n=n(\hat{x})$. In the following we will consider the above problem at a fixed boundary point $\hat{x}$ with normal $n=(1,0,0)$, i.e. we consider

$$
\begin{aligned}
v_{1} \partial_{y} \varphi+Q(\varphi) & =0 \\
\varphi(0, v) & =k(v), v_{1}>0
\end{aligned}
$$

We proceed now with the extension of a scheme derived for simple model equations in Golse/Klar [7]. 
Determination of the asymptotic value:

We consider a slightly more general equation introducing a velocity shift :

$$
\begin{aligned}
\left(v_{1}+u\right) \partial_{y} \varphi+Q(\varphi) & =0 \\
\varphi(0, v) & =k(v),\left(v_{1}+u\right)>0
\end{aligned}
$$

with $u \in \mathbb{R}$. Following, e.g. Greenberg et al. [9] this equation has a unique solution, if $u$ is bigger than 0 . If $u$ is smaller than 0 it is necessary to fix the flux

$$
\int\left(v_{1}+u\right) \varphi d v
$$

to an arbitrary constant to obtain uniqueness of the solution. We assume $u>0$. The adjoint equation to equation (20) is

$$
\begin{aligned}
-\left(v_{1}+u\right) \partial_{y} \psi+Q(\psi) & =0 \\
\psi(0, v) & =0,\left(v_{1}+u\right)<0 \\
\int\left(v_{1}+u\right) \psi(y, v) d v=1 &
\end{aligned}
$$

The reason for considering this equation will become clear in the following. The condition on the flux is needed in this case to ensure uniqueness due to the above considerations. We derive now an approximate series for $\psi$ :

Shifting velocities $v \rightarrow-v, u \rightarrow-u$ we obtain, using the definition of $Q$ and the fact that $\Phi(-v,-w)=\Phi(v, w)$

$$
\begin{aligned}
\left(v_{1}+u\right) \partial_{y} \psi+\lambda(v) \psi(v)-M(v) \int \Phi(v, w) \psi(w) d w & =0 \\
\psi(0, v) & =0,\left(v_{1}+u\right)>0 \\
\int\left(v_{1}+u\right) \psi d v=-1 . &
\end{aligned}
$$

This equation will now be solved approximately by solving macroscopic equations derived by a Chapman-Enskog type procedure: An approximate equation for

$$
\rho_{1}(y)=\int \psi(y, v) d v
$$

is obtained in the following way: Integrating (22) gives

$$
\partial_{y} \int v_{1} \psi d v+u \partial_{y} \int \psi d v=0
$$

A short calculation using the selfadjointness properties of the collision operator, see [17], gives

$$
\begin{array}{r}
\int v_{1} \psi d v=\int v_{1} \psi M M^{-1} d v=\int Q\left(h_{1}\right) \psi M^{-1} d v=\int h_{1} Q(\psi) M^{-1} d v \\
=-\partial_{y} \int h_{1}\left(v_{1}+u\right) \psi M^{-1} d v
\end{array}
$$

Approximating now $\psi$ by its mean value, i.e. $\psi \sim \rho_{1} M$ gives

$$
\int v_{1} \psi d v=-D^{u} \partial_{y} \rho_{1}
$$


with $D^{u}=\int\left(v_{1}+u\right) h_{1} d v=D+u \int h_{1} d v$. Using (23) and (24) we get

$$
u \partial_{y} \rho_{1}=D^{u} \partial_{y}^{2} \rho_{1} .
$$

This can be solved explicitely with two free parameters $A, B \in \mathbb{R}$, yielding

$$
\rho_{1}(y)=A e^{\frac{u}{D^{u}} y}+B .
$$

The first approximation $\psi_{1}$ of the solution $\psi$ of equation (22) is then obtained by approximating $\psi(w)$ in $\int \Phi(v, w) \psi(w) d w$ by $\rho_{1} M(w)$ and solving the resulting equation

$$
\begin{aligned}
\left(v_{1}+u\right) \partial_{y} \psi_{1}+\lambda(v) \psi_{1}-\rho_{1} M(v) \lambda(v) & =0 \\
\psi_{1}(0, v) & =0,\left(v_{1}+u\right)>0 .
\end{aligned}
$$

explicitely. A and B are determined by

$$
\int\left(v_{1}+u\right)\left\{\begin{array}{c}
\psi_{1}(\infty, v) \\
\psi_{1}(0, v)
\end{array}\right\} d v=-1
$$

the closest analogue to $\int\left(v_{1}+u\right) \psi d v=-1$ in $(22)$.

The $\psi$ approximation can be iterated: From (22) and (25) we obtain the equation for the remainder $\psi-\psi_{1}$ :

$$
\begin{array}{r}
\left(v_{1}+u\right) \partial_{y}\left(\psi-\psi_{1}\right)+\lambda(v)\left(\psi-\psi_{1}\right) \\
-\left[\int \Phi(v, w)\left(\psi-\psi_{1}\right)(w) d w+\int \Phi(v, w) \psi_{1}(w) d w-\rho_{1} \lambda(v)\right] M(v)=0 \\
\left(\psi-\psi_{1}\right)(0, v)=0, \quad v_{1}+u>0
\end{array}
$$

Defining $\rho_{2}:=\int\left(\psi-\psi_{1}\right) d v$ one can derive in a similar way as above an approximate equation for $\rho_{2}$, namely

$$
u \partial_{y} \rho_{2}-D^{u} \partial_{y}^{2} \rho_{2}=\int\left[\int \Phi(v, w) \psi_{1}(w) d w-\rho_{1} \lambda(v)\right] M(v) d v
$$

$\rho_{2}$ is again uniquely determined up to 2 Parameters. The approximation $\psi_{2}$ of $\psi-\psi_{1}$ is then given by the solution of

$$
\begin{gathered}
\left(v_{1}+u\right) \partial_{y} \psi_{2}+\lambda(v) \psi_{2}-\left[\lambda(v) \rho_{2}+\int \Phi(v, w) \psi_{1}(w) d w-\lambda(v) \rho_{1}\right] M(v)=0 \\
\psi_{2}(0, v)=0, \quad v_{1}+u>0 .
\end{gathered}
$$

The two parameters in the solution of (26) are determined by the condition

$$
\int\left(v_{1}+u\right)\left\{\begin{array}{c}
\psi_{2}(\infty, v) \\
\psi_{2}(0, v)
\end{array}\right\} d v=0
$$

Going on one obtains a series

$$
\psi_{1}+\psi_{2}+\cdots
$$


with

$$
\begin{gathered}
\left(v_{1}+u\right) \partial_{y} \psi_{k}+\lambda(v) \psi_{k}-\left(\lambda(v) \rho_{k}+g_{k}\right) M(v)=0, k=2 \ldots n \\
\psi_{k}(0, v)=0, v_{1}+u>0 \\
\int\left(v_{1}+u\right)\left\{\begin{array}{l}
\psi_{k}(0, v) \\
\psi_{k}(\infty, v)
\end{array}\right\}=0
\end{gathered}
$$

where

$$
g_{k}(v)=\int \Phi(v, w) \psi_{k-1}(w) d w-\lambda(v) \rho_{k-1}
$$

and

$$
u \partial_{y} \rho_{k}-D^{u} \partial_{y}^{2} \rho_{k}=\int g_{k}(v) M(v) d v .
$$

Assuming that this series converges, it can be easily seen by summing up the $\psi_{k}$ 's, that the series is equal to the solution $\psi$ of the adjoint equation (22).

Hence one only has to transform $v$ and $u$ backwards, $v \rightarrow-v, u \rightarrow-u$ to get the desired approximation of $(21), \psi(y, v)$.

Let $\varphi$ be a solution of (20) and $\psi$ one of (21), then

$$
\begin{aligned}
& \partial_{y}\left(\int\left(v_{1}+u\right) \varphi(y, v) \psi(y, v) M^{-1} d v\right) \\
& =\int\left(v_{1}+u\right)\left(\partial_{y} \varphi\right)(y, v) \psi(y, v) M^{-1} d v+\int\left(v_{1}+u\right)\left(\partial_{y} \psi\right)(y, v) \varphi(y, v) M^{-1} d v \\
& =\int Q(\psi) \varphi M^{-1} d v-\int Q(\varphi) \psi M^{-1} d v \\
& =\int Q(\psi) \varphi M^{-1} d v-\int Q(\psi) \varphi M^{-1} d v \\
& =0
\end{aligned}
$$

Using this we get

$$
\int\left(v_{1}+u\right) \varphi(\infty, v) \psi(\infty, v) M^{-1} d v=\int\left(v_{1}+u\right) \varphi(0, v) \psi(0, v) M^{-1} d v
$$

and substituting gives

$$
\int\left(v_{1}+u\right) \varphi(\infty, v) \psi(\infty, v) M^{-1} d v=\int_{\left(v_{1}+u\right)>0}\left(v_{1}+u\right) k(v) \psi(0, v) M^{-1} d v .
$$

Or with $\int\left(v_{1}+u\right) \psi(y, v) d v=1$ and since $\varphi(\infty, v) M^{-1}(v)$ is independent of $v$

$$
\varphi(\infty, v) M^{-1}(v)=\int_{\left(v_{1}+u\right)>0}\left(v_{1}+u\right) k(v) \psi(0, v) M^{-1} d v
$$

where $\psi(0, v)$ is approximated by the series

$$
\psi(0, v) \sim \psi_{1}(0, v)+\psi_{2}(0, v)+\ldots+\psi_{n}(0, v)
$$

derived above. In this way one obtains an approximation of the asymptotic value $\varphi(\infty, v) M^{-1}(v)$ of equation $(20)$. 
Taking only the first step of the above procedure, i.e., $\psi=\psi_{1}$ and the limit for $u$ tending to 0 one obtains after some calculations the following approximation for the asymptotic value $L_{\hat{x}}(k(\cdot))=\varphi\left(\infty, \alpha_{i}, v\right) M^{-1}(v)$ of equation (18):

$$
\begin{aligned}
& L_{\hat{x}}^{a}(k(\cdot))=\frac{\int_{v \cdot n>0} v \cdot n k(v) d v}{\int_{v \cdot n>0} v \cdot n M(v) d v}+ \\
& \frac{1}{D} \int_{v \cdot n>0} \frac{(v \cdot n)^{2}}{\lambda(v)}\left[k(v)-M(v) \frac{\int_{v \cdot n>0} v \cdot n k(v) d v}{\int_{v \cdot n>0} v \cdot n M(v) d v}\right] d v
\end{aligned}
$$

The first term in this formula is exactly the Maxwell-Marshak approximation. This shows that actually the Maxwell-Marshak condition is a first approximation of the asymptotic value of the solution of the halfspace problem.

\section{Approximation of the outgoing distribution:}

We consider equation (19) directly. Now the asymptotic value $\varphi(\infty, v)$ is known from the above procedure. We derive for (19) in the same way as above the macroscopic equation

$$
\partial_{y}^{2} \rho_{1}=0 .
$$

The solution is $\rho_{1}=B, B$ some constant since $\rho_{1}$ must be finite at infinity.

Then the first approximation $\varphi_{1}$ of the solution of (19) is obtained by solving

$$
\begin{aligned}
v_{1} \partial_{y} \varphi_{1}+\lambda(v) \varphi_{1}-\rho_{1} M(v) \lambda(v) & =0 \\
\varphi_{1}(0, v) & =k(v), v_{1}>0
\end{aligned}
$$

This gives

$$
\varphi_{1}(y, v)=\left\{\begin{array}{ll}
e^{-\frac{\lambda(v)}{v_{1}} y} k(v)+B\left(1-e^{-\frac{\lambda(v)}{v_{1}} y}\right) M(v), & v_{1}>0 \\
B M(v), & v_{1}<0
\end{array} .\right.
$$

To obtain $\varphi_{1}(\infty, v)=\varphi(\infty, v)$ we have to put B equal to $\varphi(\infty, v) M^{-1}$. Hence, we get for $v_{1}<0$ from (28)

$$
\varphi_{1}(0, v)=\varphi(\infty, v)
$$

This can be approximated by the method derived above. To obtain in this way the first nontrivial approximation of the outgoing distribution we have to iterate the procedure once more: Considering the equation for $\varphi-\varphi_{1}$, i.e.

$$
\begin{array}{r}
v_{1} \partial_{y}\left(\varphi-\varphi_{1}\right)+\lambda(v)\left(\varphi-\varphi_{1}\right) \\
-\left[\int \Phi(v, w)\left(\varphi-\varphi_{1}\right)(w) d w+\int \Phi(v, w) \varphi_{1}(w) d w-\lambda(v) \rho_{1}\right] M(v)=0
\end{array}
$$

one derives the second step gas dynamics equation:

$$
-D \partial_{y}^{2} \rho_{2}=\int\left[\int \Phi(v, w) \varphi_{1}(w) d w M(v)-\varphi(\infty, v) \lambda(v)\right] d v .
$$


As before $\varphi-\varphi_{1}$ is then approximated by the solution $\varphi_{2}$ of

$$
\begin{aligned}
v_{1} \partial_{y} \varphi_{2}+\lambda(v) \varphi_{2}-\left(\lambda(v) \rho_{2} M(v)\right. & \\
\left.+\int \Phi(v, w) \varphi_{1}(w) d w M(v)-\lambda(v) \varphi(\infty, v)\right) & =0 \\
\varphi_{2}(0, v) & =0, v_{1}+u>0,
\end{aligned}
$$

with $\varphi_{2}(\infty, v)=0$. Hence, solving this equation we obtain for $v_{1}<0$ the approximation $\left(\varphi_{1}+\varphi_{2}\right)(0, v)$ for the outgoing distribution $\varphi(0, v)$ of $(19)$.

Approximating the asymptotic value $\varphi\left(\infty, \alpha_{i}, v\right)$ of $(18)$ by $L_{\hat{x}}^{a}(k(\cdot))$ yields in this way the following approximation for the Albedo operator of equation (18):

$$
\begin{aligned}
& A_{\hat{x}}^{a}\left(\left.k(\cdot)\right|_{v \cdot n>0}\right)(v)=\varphi\left(0, \alpha_{i}, v\right)=L_{\hat{x}}^{a}(k(\cdot)) M(v) \\
+ & M(v) \int_{w \cdot n>0} \frac{w \cdot n}{w \cdot n \lambda(v)-v \cdot n \lambda(w)}\left[\Phi(v, w)-\frac{(w \cdot n)^{2}}{D \lambda(w)}\right] \\
& {\left[k(w)-L_{\hat{x}}^{a}(k(\cdot)) M(w)\right] d w . }
\end{aligned}
$$

for $v \cdot n<0$.

Approximation of the higher order halfspace problem (13):

We consider finally the second relevant half space problem for $\hat{\chi}\left(y, \alpha_{i}, v, t\right)$ at the interface point $\hat{x}$, namely

$$
\begin{aligned}
v \cdot n \partial_{y} \hat{\chi}+E \cdot \nabla_{v} \chi+\sum_{i=1}^{2} v \cdot \nabla_{x} \alpha_{i} \partial_{\alpha_{i}} \chi+Q(\hat{\chi}) & =0 \\
\hat{\chi}\left(0, \alpha_{i}, v, t\right) & =0, v \cdot n>0
\end{aligned}
$$

where $E=E(\hat{x}, t)$ and $n=n(\hat{x})$ and $\chi=\chi\left(y, \alpha_{i}, v, t\right)$ is the boundary layer function defined in section 4 that solves (11) with ingoing function

$$
g(\hat{x}, v, t)=f_{1}(\hat{x}, v, t)-\rho(\hat{x}, t) M(v)+O(\epsilon) .
$$

for $v \cdot n>0$. A rough approximation procedure for this equation will be given in the following:

We start by approximating $\chi\left(y, \alpha_{i}, v, t\right)$ by the above procedure. Remarking that $\chi\left(\infty, \alpha_{i}, v, t\right)$ is equal to 0 formula (28) gives

$$
\chi\left(y, \alpha_{i}, v, t\right) \sim\left\{\begin{array}{ll}
e^{-\frac{\lambda(v)}{v \cdot n} g} g(\hat{x}, v, t), & v \cdot n>0 \\
0, & v \cdot n<0
\end{array} .\right.
$$

Putting this into equation (30) we obtain an approximate equation for $\hat{\chi}\left(y, \alpha_{i}, v, t\right)$. With the same procedure as before we derive an approximate equation for $\rho\left(y, \alpha_{i}, t\right)=\int \hat{\chi}\left(y, \alpha_{i}, v, t\right) d v$ :

$$
D \partial_{y}^{2} \rho=\bar{E} \partial_{y} C\left(y, \alpha_{i}, t\right)+\sum_{i=1}^{2} \nabla_{x} \alpha_{i} \cdot \int v \partial_{\alpha_{i}} \chi\left(y, \alpha_{i}, v, t\right) d v
$$


with $D$ as before, $\bar{E}=\int E \cdot v h \cdot n d v$ and the mean value

$$
C\left(y, \alpha_{i}, t\right)=\int \chi\left(y, \alpha_{i}, v, t\right) d v=\int_{v \cdot n>0} e^{-\frac{\lambda(v)}{v \cdot n} y} g(\hat{x}, v, t) d v .
$$

Solving (32) with $\rho(0, t)=0$ and the requirement of boundedness at infinity gives $\rho\left(y, \alpha_{i}, t\right)$ and in particular the following approximation for $\hat{\chi}\left(\infty, \alpha_{i}, v, t\right)$

$$
\begin{array}{r}
\hat{\chi}^{a}\left(\infty, \alpha_{i}, v, t\right)=\rho\left(\infty, \alpha_{i}, t\right) M(v) \\
=M(v) \int_{w \cdot n>0} \frac{w \cdot n}{\lambda(w)} \frac{\bar{E}}{D}\left[f_{1}(\hat{x}, w, t)-\rho(\hat{x}, t) M(w)\right] d w \\
-\frac{1}{D} M(v) \sum_{i=1}^{2} \int_{w \cdot n>0} w \cdot \nabla_{x} \alpha_{i} \frac{(w \cdot n)^{2}}{\lambda^{2}(w)} \partial_{\alpha_{i}}\left[f_{1}(\hat{x}, w, t)-\rho(\hat{x}, t) M(w)\right] d w .
\end{array}
$$

$\hat{\chi}^{a}\left(0, \alpha_{i}, v, t\right)=\rho\left(0, \alpha_{i}, t\right) M(v)=0$ is then the first approximation of $\hat{\chi}\left(0, \alpha_{i}, v, t\right)$ for $v \cdot n<0$.

Obviously here a more sophisticated approximation could be performed at the expense of obtaining conditions that are more difficult to treat from a computational point of view.

\section{Acknowledgements}

I am grateful to F. Golse, H. Neunzert and R. Wegener for helpful discussions.

\section{References}

[1] C. Bardos, R. Santos, R. Sentis, Diffusion Approximation and Computation of the Critical Size, TAMS, Vol. 284, No. 2, p. 617, 1984

[2] A. Bensoussan, J.L. Lions, G.C. Papanicolaou, Boundary Layers and Homogenization of Transport Processes, Publ. RIMS, Kyoto Univ. 15, p. 52,

[3] J.F. Bourgat, P. Le Tallec, D. Tidriri, Y. Qiu, Numerical Coupling of Nonconservative or Kinetic Models with the Conservative Compressible NavierStokes Equations, Fifth International Symposium on Domain Decomposition methods for P.D.E., SIAM, Philadelphia 1991

[4] C. Cercignani, The Boltzmann Equation and its Applications, Springer, 19881979

[5] F. Golse, Applications of the Boltzmann Equation Within the Context of Upper Atmosphere Vehicle Aerodynamics, Computer Meth. in Engineer. and Appl. Mech. Vol. 75, p.299, 1989

[6] F. Golse, Knudsen Layers from a Computational Viewpoint, TTSP 21 (3), 211, 1992 
[7] F. Golse, A. Klar, A Numerical Method for Computing Asymptotic States and Outgoing Distributions for Kinetic Linear Half Space Problems, J. Stat. Phys. 80 (5/6), 1033-1061, 1995

[8] F. Golse, F. Poupaud, Limite fluide des equations de Boltzmann des semiconducteurs pour une statistique Fermi-Dirac, J. Asympt. Anal. 6, 135, 1992

[9] W. Greenberg, C. van der Mee, V. Protopopescu, Boundary Value Problems in Abstract Kinetic Theory, Birkhäuser, 1987

[10] R. Illner, H. Neunzert, Domain Decomposition: Linking Kinetic and Aerodynamic Descriptions, AGTM preprint 90, Kaiserslautern, 1993

[11] A. Klar, Domain Decomposition for Kinetic Problems with Nonequilibrium States, Eur. J. Mech./B Fluids, 15, 2,203-216, 1996

[12] A. Klar, Convergence of Alternating Domain Decomposition Schemes for Kinetic and Aerodynamic Equations Math. Meth. Appl. Sci., 18, 649-670, 1995

[13] E.Larsen, G.Pomraning, Initial and Boundary Conditions for Equilibrium Diffusion Theory, J. Quant. Spectr. and Radiative Transfer 36, 69, 1986

[14] S.K. Loyalka, Approximate Method in the Kinetic Theory in Phys . Fluids 11, Vol. 14, 1971

[15] A. Lukschin, H. Neunzert, J. Struckmeier, Interim Report for the Hermes Project DPH 6174/91, 1992

[16] P. Markowich, C. Ringhofer, C. Schmeiser, Semiconductor equations, Springer 1990

[17] F. Poupaud, Diffusion Approximation of the Linear Semiconductor Equation, J. Asympt. Anal. 4, 293, 1991

[18] S. Selberherr, Analysis and Simulation of Semiconductor Devices, Springer, Wien 1984

[19] Y. Sone, Asymtotic theory of a Steady Flow of a Rarefied Gas Past Bodies for Small Knudsen Numbers, in Advances in Kinetic Theory and Continuum Mechanics, Proceedings of a Symposium Held in Honour of Henri Cabannes, Eds. R. Gatignol and Soubbaramayer, Springer, Berlin 1990

[20] A. Yamnahakki, Second order boundary conditions of Drift Diffusion Equations of Semiconductors, Univ. de Nice, to appear in Math. Models and Meth. in Appl. Sci. 